\title{
Why Matrix theory works for oddly shaped membranes
}

\author{
Yonatan Zunger \\ Department of Physics \\ Stanford University \\ Stanford, CA 94305-4060 \\ (October 31, 2018)
}

\begin{abstract}
We give a simple proof of why there is a Matrix theory approximation for a membrane shaped like an arbitrary Riemann surface. As corollaries, we show that noncompact membranes cannot be approximated by matrices and that the Poisson algebra on any compact phase space is $U(\infty)$. The matrix approximation does not appear to work properly in theories such as IIB string theory or bosonic membrane theory where there is no conserved 3-form charge to which the membranes couple.
\end{abstract}

02.10.Yn,02.20.Tw,11.25.-w

\section{MATRIX THEORY AND POISSON BRACKETS}

The idea of approximating various aspects of stringy dynamics, especially the dynamics of membranes, by Matrix theories - quantum field theories in low $(0+1$ and $1+1)$ dimensions whose fields are matrix-valued - has been around for some time. In the early 1980's, Goldstone [1] and Hoppe [2] independently examined the action for a relativistic membrane and showed that, for membranes of spherical geometry, the Nambu-Goto action can be approximated to arbitrary precision by the dimensional reduction of super-Yang-Mills theory to $0+1$ dimensions, where the gauge group is $S U(N)$. As $N \rightarrow \infty$, this gauge group converged to the group of area-preserving diffeomorphism of the sphere, which is a symmetry of the original action. This action was the first and simplest example of a matrix model, and sparked the study of matrix membranes. Some of the important developments in this field since then are the extension of this result to the supersymmetric case, [3] the Matrix theory of toroidal membranes, 国 8] and the understanding, at least in theory, of how to do Matrix theory for other topologies of membrane. [9] A recent comprehensive review has been given by Taylor. [10]

The matrix approximation was immediately realized to be valuable both as a way for understanding the membranes which appeared in supergravity (and later, of course, in string theory) and as a method of regularizing field theories which is compatible with supersymmetry. Today Matrix theory (in its more sophisticated forms, relating membranes to systems of D0-branes) is one of our few nonperturbative handles on M-theory. But as we shall see below, there is a key step in the matrix membrane argument having to do with the nature of Poisson brackets on membranes which has been implicitly asserted but not yet proven. In this paper we will prove this conjecture, namely that the Poisson brackets on any Riemann surface form the algebra $U(\infty)$, and thus put the matrix membrane on a more solid footing. Phrased another way, we will show that the group of area-preserving diffeomorphisms of any Riemann surface is $S U(\infty)$; these two groups differ by a $U(1)$ factor generated by the constant functions, which generate the trivial diffeomorphism. P Phrased another way still, the group of Hamiltonian flows of a classical system with compact phase space is always $U(\infty)$. 2

\footnotetext{
${ }^{1}$ Infinitesimal area-preserving diffeomorphisms are given by $x^{i} \rightarrow x^{i}+\left\{x^{i}, \lambda\right\}$, where the braces are Poisson brackets and $\lambda$ is an arbitrary function. This follows naturally from the Liouville theorem or from demanding that the Jacobian of an arbitrary transformation be 1 . Constant functions $\lambda$ have vanishing Poisson brackets with all functions and so do not generate a diffeomorphism.

${ }^{2}$ Whenever one refers to $U(\infty)$, one must deal with the subtle issue of to which $U(\infty)$ one is referring; for example, one could define it as $\bigcup_{n} U(n)$, or as the set of limits of sequences of unitary operators of increasing dimension, which is the set of compact unitary operators on a Hilbert space. These groups have the same Lie algebra but very different topologies. The group to which we shall refer as $U(\infty)$ in this paper is the latter group, which can also be described as the group generated by infinite-dimensional clock and shift matrices. Hoppe and Schaller have given a useful discussion of how this group is indeed the group of area-preserving diffeomorphisms on the torus, as well as of the analogous groups for the noncommutative torus. [11) Harvey has recently summarized the difference between the various definitions of $U(\infty)$ and discussed these differences in the context of string theory. [12]
} 
Let us begin by demonstrating the subtlety in the usual derivation of the matrix membrane. The action for a supersymmetric membrane in type IIA string theory in the light-cone frame is 13,14

$$
S=\int d^{3} \xi \frac{1}{2} \dot{X}^{2}+\bar{\theta} \gamma_{-} \dot{\theta}-\frac{1}{4}\left\{X^{i}, X^{j}\right\}^{2}+\bar{\theta} \gamma_{-} \gamma_{i}\left\{X^{i}, \theta\right\}
$$

where the brackets represent Poisson brackets with respect to the membrane world-volume coordinates. The shape of the membrane (say, a sphere) is encoded by a vacuum expectation value (VEV) of the $X^{i}$. The observation which led to matrix membrane theory was the demonstration [1,2] that the Lie algebra of infinitesimal area-preserving diffeomorphisms on the sphere was none other than $S U(\infty)$, and therefore it could be approximated at arbitrary order by the matrices of $S U(N)$. To do this, one replaces the functions $X^{i}(\xi)$ and $\theta(\xi)$ with $S U(N)$-valued functions of time, the Poisson brackets with commutators, and the integral over the space coordinates with a trace in the matrix space. If one does this, one finds the action

$$
S=\int d t \operatorname{Tr}\left[\frac{1}{2} \dot{X}^{2}+\bar{\theta} \gamma_{i} \dot{\theta}-\frac{1}{4}\left[X^{i}, X^{j}\right]^{2}+\bar{\theta} \gamma_{-} \gamma_{i}\left[X^{i}, \theta\right]\right] .
$$

This action is immediately recognizable as the action for super-Yang-Mills theory of the gauge group $S U(N)$ dimensionally reduced to $0+1$ dimensions in temporal gauge $X^{0}=0$. The field strength in this gauge is

$$
\begin{aligned}
& F^{0 i}=\dot{X}^{i} \\
& F^{i j}=-i\left[X^{i}, X^{j}\right]
\end{aligned}
$$

and the action is

$$
S=\int d t \operatorname{Tr}\left[\frac{1}{4} F^{2}+\bar{\theta} \gamma_{-\gamma} \cdot \nabla \theta\right]
$$

This action is also the low-energy action for a system of $N$ D0-branes, where the eigenvalues of the VEV of the $X^{i}$ give the positions of the branes, and the off-diagonal elements represent amplitudes for strings to be stretched between a given pair of branes.

The problem with this argument is that it relies on the Poisson algebra of the membrane being $U(\infty)$, independent of the fields. However, the spherical geometry is encoded only by the values of the $X^{i}$; the most general set of fluctuations should include higher-genus surfaces. If the Poisson algebra were to depend on the topology of the space (and there is no a priori reason to believe that this should not be the case) then in the action (1.2) the gauge group would be a function of the gauge fields, and so this would not be a Yang-Mills action. One cannot arbitrarily restrict the fluctuations to those which preserve the Poisson algebra, since any such truncation would clearly violate unitarity; all membrane geometries might appear as intermediate states of the theory.

It is therefore crucial to understand the dependence of the Poisson algebra of a manifold on its geometry. Our key result is that the Poisson algebra of any Riemann surface (a connected, compact and orientable surface) is $U(\infty)$ ). This statement has been long conjectured but no complete proof has appeared in the literature. Using this theorem we can close the gap in the preceding argument, since now all finite-energy fluctuations about a compact background geometry should preserve compactness and thus leave the Poisson algebra fixed.

Before proving this result, let us consider the meaning of configurations which violate its three conditions. For disconnected manifolds, we will recover the usual block-diagonal behavior associated with disconnected membranes. The Poisson algebra is $U(\infty)^{n}$, with a factor for each connected component. This $U(\infty)^{n}$ is contained in $U(\infty)$, with the off-diagonal terms becoming light when two membranes are close to each other.

For noncompact manifolds, we will see that the algebra has continuously infinite dimension (this follows from the fact that one needs a continuous rather than countable basis to specify functions on a noncompact surface) and therefore no matrix approximation exists. The simplest example of this is the matrix plane, where the Poisson bracket $\{x, y\}=1$ clearly has no finite-dimensional approximations. Physically this is because a finite number of D0-branes cannot approximate an infinite surface area. In general, when one attempts to do this one can recover matrix approximations which are good for certain blocks of the matrix (those corresponding to the deep interior of the membrane) but the matrices acquire large elements at their edges. However, it appears to be possible to approximate

\footnotetext{
${ }^{3}$ It has already been shown that every membrane geometry has a matrix approximation, but the independence of this approximation on the geometry in the large- $N$ limit remains an open question. [9]
} 
these spaces using Matrix theories of spaces with boundary; this involves the introduction of boundary fields and is substantially more complicated. [15]

Nonorientable membranes such as Klein bottles cannot appear in M-theory or in type IIA theory since those membranes carry a local charge which is essentially an orientation on the world-volume. (That is, each small patch of membrane has a definite sense, and so small patches cannot be consistently glued into unorientable membrane configurations) They can appear in theories where the membranes are unstable, such as type IIB theory or bosonic membrane theory. In these theories, we need to be able to consistently treat such membranes since there is no a priori obstruction to their being in the spectrum. However, it is not clear whether these states can be adequately described by the usual matrix construction. The problem is that the Poisson brackets do not form a Lie algebra when the membrane is nonorientable. (This is because the criterion for them to form an algebra is that the symplectic form be a closed, nondegenerate 2-form. On a 2-manifold every 2-form is closed, but the existence of a nondegenerate 2-form is precisely the criterion for orientability) This means that the action cannot be written in the form (1.1) and so the meaning of the matrix approximation is unclear. It is possible that this approximation simply misses all nonorientable states; in that case there may be problems at loop level since these states should emerge as intermediate states. We therefore restrict our considerations to theories such as type IIA theory or M-theory where these states do not appear.

\section{WHY THE POISSON ALGEBRA IS ALWAYS $U(\infty)$}

We would now like to prove our main statement:

Theorem. The Poisson algebra of any compact, connected and orientable 2-manifold is $U(\infty)$.

We will prove this in two parts. Our strategy will be to define a special class of Lie algebras, the pseudocompact algebras, and show that the only simple pseudocompact algebra is $S U(\infty)$. We will then show that the Poisson algebra is $U(1)$ times a simple pseudocompact algebra. The first part of this proof is unavoidably slightly technical, but it is actually not as complicated as it may seem. We will show that one can use Dynkin diagrams to classify pseudocompact algebras in the same way that they classify compact algebras. The argument for this is very similar to the ordinary classification of finite-dimensional algebras. The only differences are (1) that a certain technical step in the proof that the simple roots are enough to reconstruct the algebra does not work in general for infinite-dimensional algebras, and we must show that it does indeed work for the pseudocompact case; (2) that all our Dynkin diagrams have infinitely many circles, and so the actual list of allowable diagrams is slightly different; and (3) that at the end, we will find four allowable Dynkin diagrams (corresponding to the four infinite classical algebras) which nontrivially correspond to the same algebra. Apart from these few points, one can read this proof with a group theory book in hand and watch an almost exactly identical development take place. The notation below has been chosen to be compatible with that found in Georgi [16] since that source is likely to be familiar to physicists. Some more technical aspects of the proof are closely related to the more generalized arguments found in Knapp. [17]

We begin with a definition: A pseudocompact Lie algebra is a Lie algebra whose Cartan-Killing metric $f^{\mathbb{A}}$ is positive definite and whose root lattice is bounded. (i.e., there is some real number greater than the magnitude of any root) For finite-dimensional algebras, the root lattice is always bounded so pseudocompactness is the same as compactness. A simple example of an algebra which satisfies the first but not the second condition, to which we will refer below, is the Virasoro algebra. From here on out we will be concerned only with countably infinite-dimensional ("countable") pseudocompact Lie algebras. We wish to prove

Proposition 1: The unique (up to isomorphism) simple, countable pseudocompact Lie algebra is $S U(\infty)$. and

Proposition 2: The Poisson algebra of any compact, connected and orientable 2-manifold (i.e. a Riemann surface) is $U(1)$ times a simple, countable pseudocompact Lie algebra.

First we will show that Dynkin diagrams are meaningful for pseudocompact algebras. Dynkin diagrams are a way of graphically representing the simple roots of the algebras, so what we must show is that the simple roots can be defined and that they contain all of the information necessary to construct the algebra. We also need to find a few properties of the simple roots which will define the conditions for a given lattice to be a valid simple root lattice, e.g. linear independence.

\footnotetext{
${ }^{4}$ The Cartan-Killing metric $\eta^{a b}=\operatorname{Tr} T^{a} T^{b}$ is the natural metric on the group manifold, and its positive-definiteness is equivalent to compactness for finite-dimensional algebras.
} 
We begin with the weights and roots. Any Lie algebra has an adjoint representation. For a countable algebra this is represented on a separable Hilbert space, and for generators $T_{a}$

$$
T_{a}\left|T_{b}\right\rangle=\left|T_{c}\right\rangle\left\langle T_{c}\left|T_{a}\right| T_{b}\right\rangle=-i f_{a b}^{c}\left|T_{c}\right\rangle=\left|\left[T_{a}, T_{b}\right]\right\rangle
$$

So let $H$ be a maximal commuting subalgebra (the Cartan subalgebra) of the algebra. Since the Cartan elements $H_{m}$ are self-adjoint and compact (this compactness following from the finite norm of the roots), the Hilbert-Schmidt theorem guarantees that their eigenvectors form a basis which simultaneously diagonalizes all of the $H_{m}$ 's. The eigenvalues are labelled by $\alpha$, (which by definition are the root vectors) and the eigenkets $\left|E_{\alpha}\right\rangle$ (being a basis for the adjoint representation) correspond to eigenoperators $E_{\alpha}$ of the Cartan subalgebra

$$
\left[H_{m}, E_{\alpha}\right]=\alpha_{m} E_{\alpha} .
$$

It is straightforward that $E_{\alpha}^{\dagger}=E_{-\alpha}$, and thus roots must occur in pairs. So to each root $\alpha$ there corresponds an $S U(2)$ subalgebra $E_{+}^{(\alpha)}=|\alpha|^{-1} E_{\alpha}, E_{-}^{(\alpha)}=|\alpha|^{-1} E_{-\alpha}, E_{3}^{(\alpha)}=|\alpha|^{-2} \alpha \cdot H$. Since the roots all have finite norm these are well-defined quantities.

Now let $|\mu\rangle$ be any state in a representation of the algebra. Its $E_{3}^{(\alpha)}$ eigenvalue is $\frac{\alpha \cdot \mu}{\alpha^{2}}$, so $\frac{2 \alpha \cdot \mu}{\alpha^{2}}$ must be an integer if the $S U(2)$ representation to which it belongs is finite. More strongly, we can say that if it belongs to a finite-dimensional representation of $S U(2)$, then

$$
\frac{2 \alpha \cdot \mu}{\alpha^{2}}=q-p
$$

where $p$ is the number of times $E_{+}^{(\alpha)}$ can act on $\mu$ without getting zero, and $q$ is the number of times $E_{-}^{(\alpha)}$ can do the same. This equation follows from the fact that $\alpha \cdot \mu / \alpha^{2}$ is the eigenvalue of $E_{3}^{(\alpha)}$ and the usual properties of $S U(2)$ representations.

One particular case of interest is the adjoint representation of the algebra. If one starts with any state $\left|E_{\beta}\right\rangle$ and acts on it repeatedly with $E_{ \pm}^{(\alpha)}$, one gets a complete representation of the $S U(2)$ associated with $\alpha$. This representation would consist of states with roots $\beta+n \alpha$ for integer $n$, and since the root lattice is bounded there cannot be more than finitely many such roots. This means that for any $\alpha$ and $\beta, 2 \alpha \cdot \beta / \alpha^{2}=q-p$ for integers $q$ and $p$.

The next step is to define simple roots. We pick a sign convention for the roots such that every nonzero root is either positive or negative, and the negative of a positive root is a negative root. Then a simple root is defined to be a positive root which is not the sum of any other positive roots. We claim that the simple roots contain enough information to reconstruct the roots, and therefore the algebra.

First, every positive root can be written as an integer linear combination of the simple roots. To see this, consider some positive root $\gamma$. If $\gamma$ is simple, the statement is trivial; otherwise $\gamma=\gamma_{1}+\gamma_{2}$ where $\gamma_{1}$ and $\gamma_{2}$ are positive. We repeat this argument indefinitely, stopping whenever a root is simple. This must terminate after no more than countably many steps, since every root has finite norm and the coefficients in the sum are all nonnegative integers.

Next, the simple roots are linearly independent. This is because the most general linear combination of simple roots can be written as a sum of terms with positive and negative coefficients;

$$
\gamma=\sum_{\alpha} \mu_{\alpha} \alpha-\sum_{\alpha} \nu_{\alpha} \alpha
$$

where the $\mu_{\alpha}$ and the $\nu_{\alpha}$ are all nonnegative integers, and for any given $\alpha$ no more than one of $\mu_{\alpha}$ and $\nu_{\alpha}$ is nonzero. Then

$$
|\gamma|^{2}=|\mu|^{2}+|\nu|^{2}-2 \sum_{\alpha \neq \beta} \mu_{\alpha} \nu_{\beta} \alpha \cdot \beta
$$

However, $\alpha \cdot \beta$ must be nonpositive. This can be shown as follows. First, for any two distinct simple roots $\alpha$ and $\beta$, $\alpha-\beta$ is not a root, since if it were then either $\alpha-\beta>0$, in which case $\alpha=(\alpha-\beta)+\beta$, or $\beta-\alpha>0$, in which case $\beta=(\beta-\alpha)+\alpha$, violating the simplicity of $\alpha$ and $\beta$ in either case. This means that $E_{-\alpha}\left|E_{\beta}\right\rangle \propto\left|E_{\beta-\alpha}\right\rangle=0$, so $2 \alpha \cdot \beta / \alpha^{2}=-p \leq 0$. (Since the relevant $q$ is by definition zero) This means that the third term in (2.5) is nonnegative, so $|\gamma|^{2} \geq 0$. By positivity of the metric, this means that it vanishes only for $\gamma=0$, so no (finite or infinite) linear combination of the simple roots can vanish.

This therefore means that the simple roots form a basis for the set of all roots. Since there are countably many roots and the root lattice is bounded, the root lattice has countably infinite dimension, and so the number of simple roots is countably infinite as well. 
Now at last we can construct the roots from the simple roots. Any positive root can be written as

$$
\phi=\sum k_{\alpha} \alpha
$$

for positive integer coefficients $k_{\alpha}$. We define the level of the root to be the sum of these coefficients, and find the roots by induction on the level. The set of level- 1 roots is just the set of simple roots. Then given the set of level- $(\ell-1)$ roots, the level- $\ell$ roots are given by

$$
\left|E_{\phi_{\ell}}\right\rangle=E_{\alpha}\left|E_{\phi_{\ell-1}}\right\rangle
$$

for every $\alpha$ and $\phi_{\ell}$ for which the right-hand side is not zero. That can be determined using

$$
\frac{2 \alpha \cdot \phi_{\ell-1}}{\alpha^{2}}=q-p
$$

where $p$ is the number of times $\left|E_{\phi_{\ell-1}}\right\rangle$ can be acted on by $E_{\alpha}$ without reaching zero. $\phi_{\ell-1}+\alpha$ is a root iff $p>0$. But $q$ is known since we know how $\left|E_{\phi_{\ell-1}}\right\rangle$ was built out of the simple roots, so $p$ can be evaluated from (2.8) and (2.7) generates the set of level- $\ell$ roots.

This can only fail if some $\left|E_{\phi_{\ell}}\right\rangle$ cannot be written in the form (2.7). The fact that this is impossible can be seen as follows. Knapp corollary 2.37 states that whenever no multiple other than 1, 0 or -1 of any root is a root (which is always true for finite-dimensional algebras; cf. Knapp proposition 2.21) the operator $E_{\alpha} E_{-\alpha}$ is nondegenerate for all $\alpha$. Pseudocompact algebras must satisfy this condition, since for any root $\alpha$ the set $\left\{\alpha \cdot H, E_{n \alpha}\right\}$ for all integers $n$ for which $n \alpha$ is a root is a subalgebra of our algebra, and by boundedness of the root lattice it is finite-dimensional. Then the usual argument that in a finite-dimensional algebra no multiple of a root greater than 1 is a root applies, and so $n \alpha$ cannot be a root for $|n|>1$.

This tells us that $\left|E_{\phi_{\ell}}\right\rangle$ is annihilated by every $E_{-\alpha}$, since if it were not then $E_{-\alpha}\left|E_{\phi_{\ell}}\right\rangle$ would be a level- $(\ell-1)$ root and acting on it with $E_{\alpha}$ would give $\left|E_{\phi_{\ell}}\right\rangle$ in the form (2.7).5 (It would not be zero since $E_{\alpha} E_{-\alpha}$ is nondegenerate) Therefore for each simple root $\alpha, E_{-\alpha}\left|E_{\phi_{\ell}}\right\rangle=0$ and so from our usual formula $2 \alpha \cdot \phi_{\ell} / \alpha^{2}=-p \leq 0$. This means that $\phi_{\ell}^{2}=\sum k_{\alpha} \alpha \cdot \phi_{\ell} \leq 0$ and (by positive definiteness) $\phi_{\ell}=0$, a contradiction. Thus every positive root can be written in the form (2.7) and so is reached by this inductive process.

Therefore the simple roots carry all of the information needed to reconstruct the algebra, and thus there is a one-to-one correspondence between simple root lattices and countable pseudocompact Lie algebras. The simple roots must be linearly independent, the angles between them must satisfy $\cos \theta_{\alpha \beta}=-\sqrt{p p^{\prime}} / 2=0,1 / 2,1 / \sqrt{2}$, or $\sqrt{3} / 2$, and their length ratios must satisfy $|\alpha|^{2} /|\beta|^{2}=p / p^{\prime}$. We can therefore introduce Dynkin diagrams to represent the simple root lattices, drawing a circle for each simple root and joining the circles $\alpha$ and $\beta$ by $4 \cos ^{2} \theta_{\alpha \beta}$ lines. The set of countable pseudocompact Lie algebras is isomorphic to the set of Dynkin diagrams with countably many circles.

We can now repeat the ordinary classification of Dynkin diagrams. Simple algebras correspond to connected diagrams since if the simple roots decompose into two orthogonal subsets (and thus two networks with $\cos ^{2} \theta_{\alpha \alpha^{\prime}}=0$ between elements of the two subsets) the corresponding ladder operators commute and thus the algebra factors.

Linear independence implies the following five standard lemmas: (The proofs are given in Georgi chapter 20 or Knapp chapter 2)

Lemma 0. Every subdiagram of a Dynkin diagram is itself a consistent Dynkin diagram.

Lemma 1. The only Dynkin diagrams with three root vectors are those given in figure 1 .

Lemma 2. If a diagram contains two vectors connected by a single line, the diagram obtained by shrinking the line away and merging the two vectors into a single circle represents another consistent diagram.

Lemma 3. In figure 2, if the first configuration is a consistent diagram, then the second is as well.

Lemma 4. No diagram contains any of the subdiagrams in figure 3 .

\footnotetext{
${ }^{5}$ For algebras which are not pseudocompact, this argument does not hold. For example, the Virasoro algebra $\left[L_{m}, L_{n}\right]=$ $i(m+n) L_{m+n}$ has one element, $L_{0}$, in its Cartan subalgebra, and root vectors $E_{n}=L_{-n}$. Its root lattice is unbounded, and so for example $E_{1} E_{-1}$ is degenerate; $L_{-1} L_{1}\left|L_{-2}\right\rangle=\left|\left[L_{-1},\left[L_{1}, L_{-2}\right]\right]\right\rangle=0$. As a result the usual root-building algorithm fails to find the positive root $L_{-2}$ and so this algebra cannot be reconstructed from its simple roots alone. This is why boundedness of the root lattice is so important.
} 

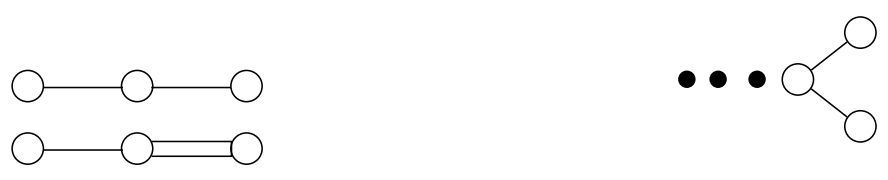

FIG. 1. Lemma 1

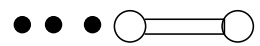

FIG. 2. Lemma 3

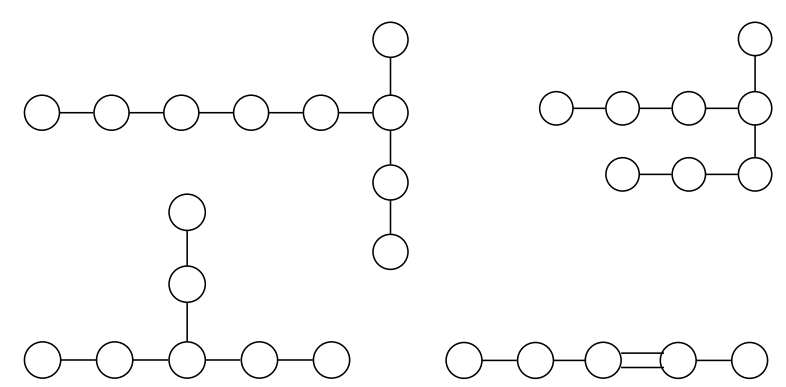

FIG. 3. Lemma 4

These do not rule out some nontrivial infinite diagrams, since lemma 2 only allows us to contract finite chains of singly-connected roots. (There may be subtleties for infinite chains) We therefore need to prove the additional lemma

Lemma 5. The diagrams shown in figure 1 are not consistent.

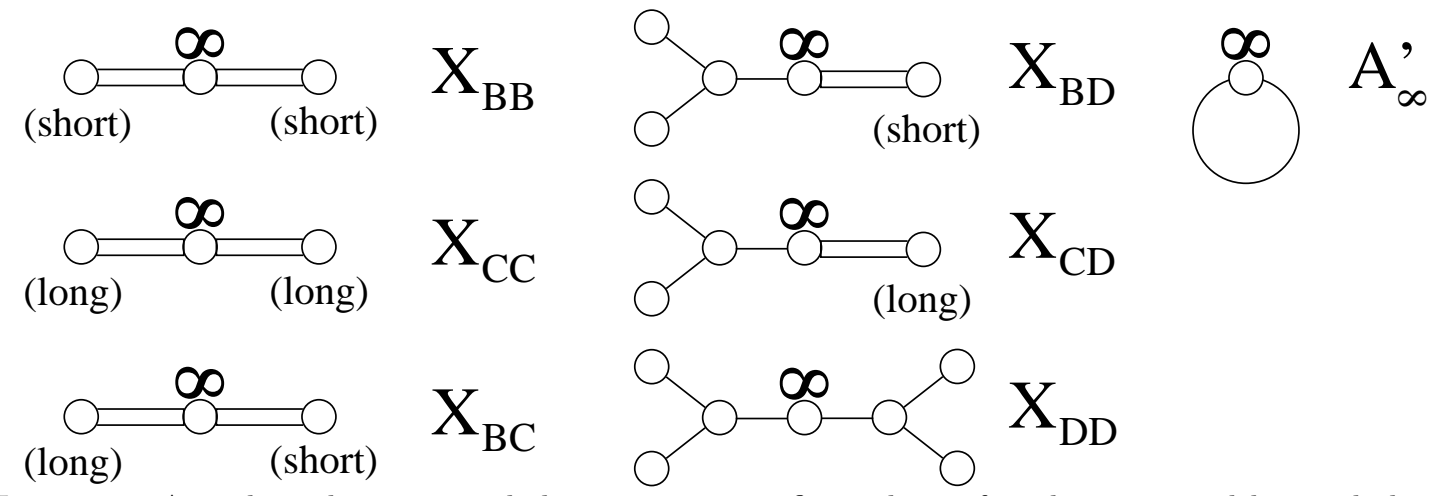

FIG. 4. Lemma 5. A circle with an $\infty$ symbol represents an infinite chain of circles connected by single lines; $A_{\infty}^{\prime}$ is an infinite loop.

We give an explicit proof for $X_{B B}$; the remaining cases are essentially identical. If we had a rank- $(n+2)$ version of this algebra, with the infinite chain replaced by a chain of length $n$, then from the diagram we can read

$$
\alpha_{0}^{2}=\alpha_{n+1}^{2}=1 ; \alpha_{i}^{2}=2
$$

where $i=1 \ldots n$, and

$$
\alpha_{i} \cdot \alpha_{i \pm 1}=\alpha_{0} \cdot \alpha_{1}=\alpha_{n} \cdot \alpha_{n+1}=-1
$$

with all other products vanishing. Therefore the linear combination $\sum \alpha$ is orthogonal to every root, since

$$
\begin{aligned}
& \left(\sum \alpha\right) \cdot \alpha_{1}=\alpha_{1}^{2}+\alpha_{1} \cdot \alpha_{2}=0 \\
& \left(\sum \alpha\right) \cdot \alpha_{2}=\alpha_{2}^{2}+\alpha_{1} \cdot \alpha_{2}+\alpha_{3} \cdot \alpha_{2}=0
\end{aligned}
$$

etc. Since the simple roots are a basis, this means that $\sum \alpha=0$ and thus these roots fail to be linearly independent. Since this can be done for any $n$, this combination is an obvious ansatz for the infinite case $X_{B B}$. There is no subtlety in the limit $n \rightarrow \infty$ because the criterion for linear independence of the simple roots does not rely on the finiteness of the linear combination. A similar calculation follows for each of the remaining algebras. 


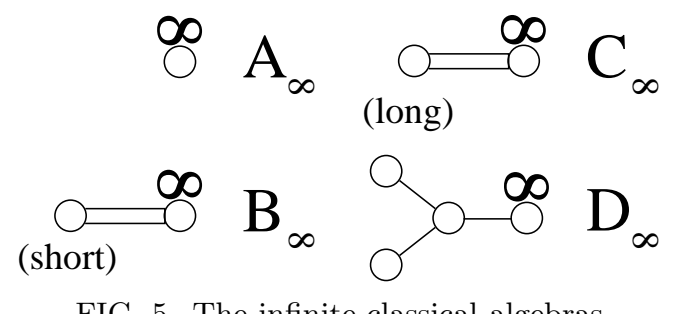

FIG. 5. The infinite classical algebras

Therefore the only consistent pseudocompact Lie algebras are the four infinite classical algebras. (Figure 5 ) We will now show that these four algebras are actually identical. (The following can also be seen explicitly by writing the various algebras in a basis of clock and shift matrices; a complete discussion has been given by Fairlie et al. [18]) We need for this a standard lemma about algebras:

Lemma. Let $U_{n}$ and $V_{n}$ be two sequences of algebras which have inductive limits $U$ and $V$, respectively. If for each $n, U_{n} \subset V_{n+1}$ and $V_{n} \subset U_{n}$, then $U \cong V$.6

The simplest application of this lemma is to show that $B_{\infty}=D_{\infty}$. These two algebras are the inductive limits of the sequences $B_{n}=S O(2 n+1)$ and $D_{n}=S O(2 n)$, respectively. Clearly $S O(2 n) \subset S O(2 n+1)$ and $S O(2 n+1) \subset$ $S O(2 n+2)$, and therefore the two limits must be equal. This is just the statement that $\lim _{n \rightarrow \infty} S O(n)$ is the same whether one progresses by even or odd $n$.

Similarly, we can show that $B_{\infty}=A_{\infty}$. For every $n, S O(n) \subset S U(n)$ since every orthogonal matrix is unitary. (This is because every real number is also a complex number) It is also well-known (and clear from Dynkin diagrams) that for each $n, S U(n) \subset S O(2 n)$. Thus using the sequences $U_{n}=S U\left(2^{n}\right)$ and $V_{n}=S O\left(2^{n}\right)$, the claim is proved.

Finally, we can show that $A_{\infty}=C_{\infty}$. For every $n, S U(n) \subset S p(2 n)$, since every complex number is also a quaternion. Similarly $S p(2 n) \subset S U(2 n)$ since the generators of each $S p$ group are Hermitian and therefore are also generators of the $S U$ group of the same dimension. Therefore all four of the classical infinite algebras are isomorphic, and so we have proven the first proposition.

Now we turn to the second proposition. Since our manifold is orientable, it automatically has a Poisson structure defined by setting its symplectic form equal to its volume form. The dimension of the Poisson algebra is countably infinite by Fourier's theorem; namely, the set of functions on a compact manifold has a countable basis.7 The elements of the Poisson algebra must be smooth functions, since functions of finite differentiability class would not be closed under the Poisson bracket.

We can see that the algebra has a $U(1)$ factor since the set of constant functions is 1-dimensional and has vanishing Poisson bracket with all functions. That the algebra is otherwise simple can be seen as follows. There is a one-to-one correspondence between invariant subalgebras of the Poisson algebra $P$ and projections on the algebra, that is to say mappings $\phi: P \rightarrow P$ which preserve the Lie bracket and satisfy $\phi^{2}=\phi$. Explicitly, for any invariant subalgebra $H$, the mapping $\phi_{H}(p)=p$ if $p \in H$ and 0 if $p \notin H$ is a projection; conversely, for any projection $\phi$ and $a, b \in P$ such that $\phi(a)=a$ and $\phi(b)=0$, (the only eigenvalues of a projection are zero and one, so every element of $P$ satisfies one or the other condition) then $\phi([a, b])=[\phi(a), \phi(b)]=0$, and $(1-\phi)([a, b])=[(1-\phi)(a),(1-\phi)(b)]=0$, so $[a, b]=0$ and thus the algebra factors into $P=\phi(P) \oplus(1-\phi)(P)$. Projections on the Poisson algebra are clearly also projections on the algebra of smooth functions under multiplication, and vice versa. (After all, both are algebras of functions; only their product rule is different) A projection in the algebra of smooth functions is a function which is everywhere either zero or one, and so is piecewise constant, and so the number of invariant subalgebras of $P$ is equal to the number of piecewise constant smooth functions on the manifold, that is to say the number of components. For a connected manifold, which is the case of interest to us, the only piecewise constant function is the constant function, corresponding to the invariant $U(1)$ subalgebra noted above.

Finally, we must prove that the algebra is pseudocompact, i.e. that its Cartan-Killing metric is positive definite and that its root lattice is bounded. This is straightforward since it is easy to show that the Poisson algebra must be

\footnotetext{
${ }^{6} \mathrm{~A}$ simple proof of this using commutative diagrams is given in lemma L.1.5 of Wegge-Olsen. 19

7 The contrapositive of this theorem gives us an immediate and important result, alluded to above, that if the membrane were unbounded (e.g. as the plane) then its Poisson algebra would have uncountably infinite dimension. The remainder of the proof below will still apply, telling us that the Poisson algebra is still $U(1)$ times a simple pseudocompact algebra, but we do not have any useful results on the classification of these much larger algebras. But these algebras clearly cannot be approximated by matrix algebras, since a sequence of finite-dimensional objects cannot converge to one of uncountable dimension.
} 
a subgroup of $U(\infty)$, and any subalgebra of a pseudocompact algebra must be pseudocompact as well. To see this, we note that for any real-valued function, the generator associated to it is

$$
T_{f}=i\{f, \cdot\}=i \Omega^{i j} \partial_{i} f \partial_{j}
$$

which is Hermitian. Since all the generators are Hermitian and the algebra is countable, the algebra must be a subalgebra of the algebra of all Hermitian, countable matrices, which is precisely $U(\infty)$. This completes the proof of proposition 2, which together with proposition 1 proves our theorem.

It is interesting that the proof of proposition 2 did not require that the manifold be 2-dimensional at any point; in fact, it applies equally well to any compact symplectic manifold. These manifolds are not the phase spaces of ordinary systems, since momentum is generally not constrained, but they may be natural phase spaces for systems which exhibit UV/IR dualities of various sorts, including T-duality.

We have therefore shown that there is a systematic matrix approximation to the theory of compact relativistic orientable membranes, in the sense that there is an infinite sequence of classical Lagrangians which converges in the large- $N$ limit to the Lagrangian (1.1). We have also seen that membrane theories which do not satisfy these constraints - theories of infinite branes or theories which allow nonorientable branes - do not seem to have a meaningful matrix approximation, or at least not one by dimensionally reduced Yang-Mills theories. This latter conclusion may be interesting from the perspective of the bosonic M-theory recently proposed by Horowitz and Susskind. [20] Applied to traditional M-theory, this is further evidence for the hypothesis that the matrix model carries the full structure of the theory.

The author would like to thank Savas Dimopoulos, Simeon Hellerman, Matthew Kleban, Steve Shenker and Leonard Susskind for helpful comments. This work was supported by the National Science Foundation under grant number PHY-9870015.

[1] Goldstone, J., 1982, unpublished.

[2] Hoppe, J., 1982, Ph.D. thesis. (Massachusetts Institute of Technology); Elem. Part. Res. J. 3, 80 (1989/90)

[3] B. de Wit, J. Hoppe and H. Nicolai, Nucl. Phys. B 305, 545 (1988).

[4] D. B. Fairlie, P. Fletcher and C. K. Zachos, Phys. Lett. B 218, 203 (1989).

[5] E. G. Floratos, Phys. Lett. B 228, 335 (1989).

[6] G. Landi, F. Lizzi and R. J. Szabo, hep-th/9912130

[7] L. Alvarez-Gaume and S. R. Wadia, hep-th/0006219.

[8] T. Krajewski and R. Wulkenhaar, Int. J. Mod. Phys. A15, 1011 (2000) hep-th/9903187.

[9] M. Bordemann, E. Meinrenken and M. Schlichenmaier, Commun. Math. Phys. 165, 281 (1994) hep-th/9309134].

[10] W. Taylor, hep-th/0101126.

[11] J. Hoppe and P. Schaller, Phys. Lett. B 237, 407 (1990).

[12] J. A. Harvey, hep-th/0105242.

[13] M. Claudson and M. B. Halpern, Nucl. Phys. B 250, 689 (1985).

[14] E. Bergshoeff, E. Sezgin and P. K. Townsend, Phys. Lett. B 189, 75 (1987).

[15] A. P. Polychronakos, JHEP 0104, 011 (2001) [hep-th/0103013].

[16] H. Georgi, Lie Algebras in Particle Physics, (Perseus Books, Reading, Mass. 1999)

[17] A. W. Knapp, Lie Groups Beyond an Introduction, Progress in Mathematics, v. 140, (Birkhäuser, Boston, Mass. 1996)

[18] D. B. Fairlie, P. Fletcher and C. K. Zachos, J. Math. Phys. 31, 1088 (1990).

[19] N. E. Wegge-Olsen, K-Theory and $C^{\star}$-Algebras, (Oxford Science Publications, 1993)

[20] G. T. Horowitz and L. Susskind, hep-th/0012037. 